\title{
Sistema de identificación de intención de movimiento para el control mioeléctrico de una prótesis de mano robótica' ${ }^{1}$ Movement Intention Detection System for Myoelectric Control of a Prosthetic Robotic Hand ${ }^{2}$
}

César Augusto Quinayás-Burgos ${ }^{3}$

Carlos Alberto Gaviria-López ${ }^{4}$

doi: 10.11144/Javeriana.iyu19-1.siim

Como citar este artículo:

C. A. Quinayás-Burgos y C. A. Gaviria-López, "Sistema de identificación de intención de movimiento para el control mioeléctrico de una prótesis de mano robótica", Ing. Univ., vol. 19, no. 1, pp. 27-50, Ene.-Jun., 2015. http://dx.doi.org/10.11144/ Javeriana.iyu19-1.siim

\footnotetext{
' Fecha de recepción: 8 de agosto de 2014. Fecha de aceptación: 24 de noviembre de 2014. Este artículo se deriva de un proyecto de investigación denominado "Contribución al desarrollo y control de prótesis de mano", desarrollado por el grupo de investigación en Automática Industrial de la Universidad del Cauca, Popayán, Colombia.

${ }^{2}$ Submitted on: August $8^{\text {th }}, 2014$. Accepted on: November $24^{\text {th }}$, 2014. This article is derived from a research project entitled "Contribution to the development and control of hand prosthesis". Developed by the Industrial Automation Research Group of the Universidad del Cauca, Popayán, Colombia.

${ }^{3}$ Ingeniero físico. Magíster en Automática, Universidad del Cauca, Popayán, Colombia. PhD (c) en Ciencias de la Electrónica, Universidad del Cauca, Popayán, Colombia. Correo electrónico: cquinayas@unicauca.edu.co.

${ }^{4}$ Ingeniero electrónico, Universidad del Cauca, Popayán, Colombia. Doctor en Automática Avanzada y Robótica, Universidad Politécnica de Cataluña, Barcelona, España. Profesor, Departamento de Electrónica, Instrumentación y Control. Universidad del Cauca. Correo electrónico: cgaviria@unicauca.edu.co.
} 


\section{Resumen}

Este artículo presenta un sistema embebido que detecta en tiempo real la intención de movimiento para el control de un prototipo de prótesis de mano robótica. El trabajo muestra que usando características temporales de cálculo simple es posible obtener un agrupamiento de vectores lo suficientemente discriminante como para que se puedan usar clasificadores de patrones muy simples. Así, en este trabajo se propone un clasificador basado en la mínima distancia al centroide de los grupos que caracterizan los movimientos que se van a identificar, modificando el conocido algoritmo $k$ vecinos más próximos para sacar mayor provecho de la fase de entrenamiento del clasificador en la fase de clasificación y obtener respuesta en tiempo real. Se presentan resultados de clasificación de intención de movimiento, obtenidos usando el porcentaje de éxito como medida de efectividad, al realizar pruebas sobre tres sujetos con músculos sanos. Los resultados experimentales muestran que el sistema puede ser efectivo para el control de ejecución de cuatro primitivas motoras de un prototipo de prótesis de mano robótica.

\section{Palabras clave}

Electromiografía (EMG); reconocimiento de intención de movimiento; K-vecinos más próximos; prótesis de mano robóticas

\section{Abstract}

This paper presents an embedded system that detects in real time the movement intention to control a prosthetic hand. This work shows that using temporal characteristics of simple calculation can provide subsets of feature vectors discernible enough as to use simple pattern classifiers. Thus, this paper proposes a classifier, which is based on the minimum distance from the centroid of the groups characterizing the movements to identify, by modifying the known algorithm K-nearest neighbors. Movement intention classification results obtained from the developed system are shown: using the percentage of success as an effectiveness measurement, by conducting tests over three persons with healthy muscles. The experimental results show that this system can be used effectively for the control of execution of four motor primitives on a prosthetic robotic hand.

\section{Keywords}

Electromyography (EMG); pattern recognition; K-nearest neighbors; prosthetic robotic hand 


\section{Introducción}

El control mioeléctrico de prótesis activas de mano ha sido utilizado en los desarrollos comerciales y de investigación hace 20 años, aproximadamente. Se distinguen dos métodos para captar las señales: no invasivo, si los electrodos se ubican sobre la superficie de músculos activos en el brazo u otra parte del cuerpo del paciente, o invasivo, si se ubican en el interior de los músculos. A pesar de las ventajas de las técnicas invasivas, aún no se ha presentado un modelo que alcance el éxito comercial. La potencialidad de los métodos no invasivos sigue siendo de interés, aun en el control de prótesis de alto nivel de destreza, como son las manos Shadow [1], BeBionic [2] o SmartHand [3].

Las prótesis activas comerciales contemplan pocos grados de libertad. La mano Otto Bock DMC Plus [4], que es una de las soluciones comerciales más representativas, solo puede abrir la mano, hacer un agarre de poder y hacer la pronación o supinación de la muñeca. En tales casos, el procesamiento de las señales electromiográficas (EMG) puede ser tan básico como una simple detección de nivel, y es suficiente con la ubicación de dos electrodos.

Dado el poco confort que se obtiene con las prótesis comerciales, hay un gran interés científico en aumentar el grado de libertad de las prótesis, lo que impone mayores desafíos al procesamiento de las señales EMG [4], porque no es práctico usar un nuevo electrodo EMG aislado para detectar cada nuevo grado de libertad. Para resolver este problema, han emergido dos caminos de investigación: usar técnicas de reconocimiento de patrones [5] o usar estimación de fuerza basada en modelo [6].

El campo del reconocimiento de patrones tiene un amplio recorrido histórico, especialmente por sus aplicaciones en técnicas de visión por computador, razón por la cual es frecuente encontrar aportes al problema desde ese enfoque. En este campo, cada sensor EMG origina un canal de señales, las cuales, luego de un procesamiento de adecuación, permiten extraer características del dominio del tiempo, de la frecuencia o de los dos. Combinando diferentes tipos de características, y la información de varios canales, se conforma un vector de características 
a partir del cual puede identificarse la intención de movimiento usando alguna de las numerosas técnicas de clasificación reportadas en la literatura [7].

Por otro lado, debido a que las señales EMG son muy susceptibles de corrupción por efecto de campos electromagnéticos cercanos, e incluso por cambios fisiológicos del paciente, la mejora de la precisión de los algoritmos para detectar la intención de movimiento también ha sido un tema de investigación de interés hasta la fecha. En este campo, los aportes en el control de prótesis avanzadas se pueden clasificar en tres grandes grupos: 1) aportes en cuanto al hardware de adquisición y procesamiento de señales [3], 2) aportes en cuanto a combinaciones de características extraídas de las señales EMG que proporcionen vectores de características que sean discriminantes [8], [9], y 3) aportes acerca de los métodos de clasificación desde múltiples enfoques, como: redes neuronales artificiales (RNN) [10], [11], lógica difusa (FL) [12], análisis discriminante lineal (LDA) [13], máquinas de soporte vectorial (SVM) [14], k vecinos próximos (k-NN) [15].

No obstante la gran variedad de aportes en estos grupos de campos de acción, a mayor complejidad de las características y de los algoritmos de clasificación seleccionados, mayor capacidad de cómputo requerida. Por tal razón, muchas de las plataformas experimentales usadas para validación emplean computadores personales como hardware de desarrollo [16], [17].

Para el caso particular del control de prótesis robóticas, el reconocimiento de la intención de movimiento debe calcularse en un sistema incrustado por las limitaciones de tamaño físico, peso y necesidad de procesamiento en tiempo real [18], [19], por lo cual muchos de los aportes reportados no son prácticos en tales sistemas.

Luego, a pesar de las numerosas contribuciones existentes sobre procesamiento de señales EMG para el control de prótesis activas de varios grados de libertad, la situación actual es que comercialmente no se han explotado las potencialidades de esas contribuciones, por lo cual las prótesis comerciales ofrecen funcionalidades limitadas para la población con discapacidades tales como la amputación del antebrazo (transradial).

La principal razón para esta situación es el compromiso entre el desempeño y la aplicación práctica de las soluciones. Por ello, en el grupo de Investigación en Automática Industrial de la Universidad del Cauca, se viene realizando un trabajo tendiente a contribuir a la solución del problema descrito, para lo cual se han abordado dos frentes.

El primero es la construcción de un prototipo de prótesis activa de mano de nueve grados de libertad con propósitos de investigación, cuyo diseño fue 
propuesto en 2010 [20] y sobre el cual se han desarrollado a la fecha algunas estrategias de control cuyo punto de partida es la detección de intención de movimiento desde señales EMG.

El segundo frente (objeto de este artículo) propone y desarrolla un sistema de reconocimiento de intención de movimiento no invasivo a partir de señales EMG. El sistema propuesto tiene las siguientes capacidades: 1) ser implementado en un sistema incrustado, 2) obtener resultados de clasificación en tiempo real para gobernar el prototipo de mano y 3) discriminar cuatro intenciones de movimiento usando como máximo dos electrodos EMG.

El elemento diferenciador de este trabajo frente a los aportes encontrados en la literatura es la propuesta de un clasificador simple que saca provecho de la información de entrenamiento fuera de línea del clasificador, con lo cual resultan un algoritmo en línea muy eficiente en tiempo de cálculo y tamaño de código. El algoritmo de clasificación propuesto combina el método de agrupación k-means con el método de clasificación k-NN. La simplicidad del clasificador propuesto hace que su eficiencia dependa en gran medida de la capacidad discriminante de los vectores de características obtenidos, lo que implica que debe hacerse una identificación individual para cada usuario, determinando buenas ubicaciones de electrodos; esta es la principal limitación del clasificador.

El sistema de identificación construido permite que el prototipo de prótesis de mano pueda realizar en tiempo real cuatro posturas comunes en la vida diaria: agarre de poder, mano abierta, pinza gruesa y volver a la posición de mano en reposo, lo que ampliaría potencialmente las capacidades de una prótesis comercial.

El sistema no ha sido probado en pacientes con amputación de mano- este es un trabajo para realizar en el futuropenden de poder encontrar músculos con actividad mioeléctrica en cada paciente y hacer un proceso individual de entrenamiento del clasificador.

Aunque el sistema se utiliza para gobernar cuatro posturas de un prototipo de prótesis de mano aplicable a pacientes con amputación transradial, podría ser utilizado por cualquier sistema automático que deba ser gobernado por una persona y que realice hasta cuatro operaciones diferentes.

\section{Materiales y métodos}

Si bien el diseño del prototipo de prótesis de mano usado para verificar el resultado del sistema propuesto no es objeto de este artículo, es preciso explicar que el diseño del prototipo de prótesis obedece a la premisa de devolver a los potenciales usuarios 
algunas facilidades para asir objetos que no proveen las prótesis comerciales, sin aumentar el número de electrodos que se usan para su control.

El prototipo está constituido por tres dedos: pulgar, índice y medio. Su tamaño es aproximadamente el de una mano adulta y pesa alrededor de $0,30 \mathrm{~kg}$. Cada uno de los tres dedos posee dos articulaciones, es decir, los dedos se accionan por seis motores DC, cinco de los cuales son empleados para la flexoextensión independiente de los dedos y uno para posición del dedo pulgar. Se utilizan sensores de posición en las articulaciones y sensores de fuerza en la yema de los dedos para el control de seguimiento de trayectorias y de fuerza.

La estrategia de control del prototipo consiste en el seguimiento de trayectorias articulares para lograr cuatro posturas deseadas en una fase que en la literatura se denomina preshaping; después de detectar el contacto entre los dedos y el objeto que se va a asir, el controlador conmuta a una fase de control de fuerza, denominada grasping.

Se seleccionaron cuatro posturas que se usan cotidianamente: mano en reposo, mano abierta, pinza gruesa y agarre de poder, como se muestra en la figura 1.

La articulación del sistema EMG descrito en este artículo con el control del prototipo se realiza en la fase de preshaping, en la cual, una vez identificada una nueva etiqueta de intención de movimiento, el control selecciona el conjunto de trayectorias articulares de la mano apropiado para el logro de esa postura. De este modo, el control de los seis grados de libertad del prototipo se hace en función de la postura que desea realizar el usuario, y no directamente de la información de cada canal EMG, con lo que pocos canales EMG permiten controlar múltiples grados de libertad.

El sistema propuesto se representa de forma esquemática en la figura 2 . Consta de un hardware para adquisición y procesamiento de información y de un software para detección de intención de movimiento. El sistema se compone de tres subsistemas: 1) sistema de acondicionamiento de las señales EMG, el cual adquiere, amplifica y filtra las señales que captan los dos electrodos; 2) sistema de procesamiento en línea de las señales EMG, el cual proporciona, por un lado, tres características del dominio del tiempo extraídas de las señales EMG y, por el otro, una etiqueta que identifica una de las cuatro posturas que se pueden realizar con el prototipo de prótesis; y 3) sistema de procesamiento fuera de línea, utilizado solo en la fase de entrenamiento del clasificador, el cual permite ajustar y programar los parámetros del clasificador para el sistema de procesamiento en línea. 
Figura 1. Posturas motoras: a) Prototipo de prótesis de mano y mano humana; b) mano en reposo; c) mano abierta; d) pinza gruesa; e) agarre de poder

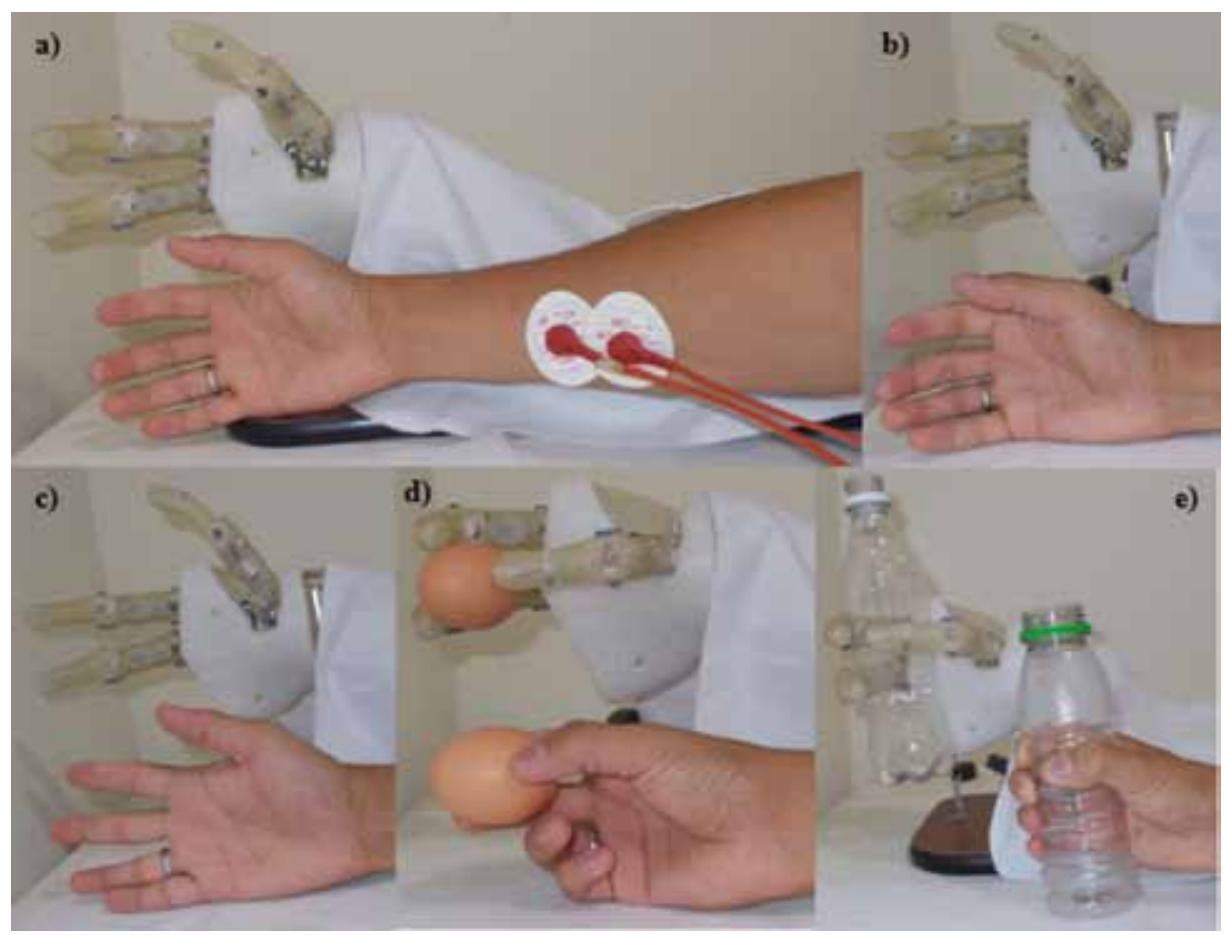

Fuente: presentación propia de los autores

Los siguientes apartados de este artículo presentan detalles de la construcción y el funcionamiento de esos sistemas y en ellos se describen los nombres de las señales utilizadas en la figura 2.

Figura 2. Esquema del sistema de reconocimiento de intención de movimiento

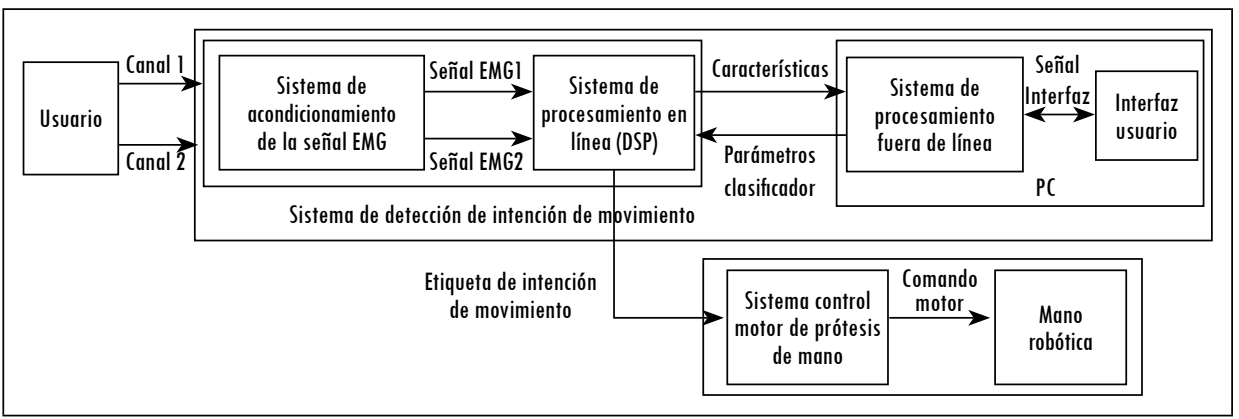

Fuente: presentación propia de los autores

Ing. Univ. Bogotá (Colombia), 19 (1): 27-50, enero-junio de 2015 


\subsection{Hardware del sistema de detección de intención de movimiento}

La señal EMG es una medida de la actividad eléctrica en el músculo. El sistema nervioso central controla dicha actividad eléctrica, la cual es producto de la contracción muscular. Su magnitud típica está entre $0 \mathrm{mV}$ y $6 \mathrm{mV}$, y normalmente está contaminada por varias fuentes de ruido, como la interferencia electromagnética de $60 \mathrm{~Hz}$ de las líneas de alimentación eléctrica, los niveles DC introducidos por los amplificadores operacionales, la interferencia electromagnética de artefactos eléctricos que se inducen en los cables que conectan el electrodo al amplificador, la interfaz entre la superficie de detección del electrodo y la piel; estos factores pueden tener efecto sobre el resultado de la extracción de características de la señal [21].

En este estudio, las señales EMG fueron captadas utilizando electrodos superficiales tipo $\mathrm{Ag} / \mathrm{AgCl}$ (3M Health Care, Alemania), posicionados sobre dos músculos: el extensor superficial (canal 1) y el flexor superficial (canal 2) del antebrazo derecho del sujeto, como se muestra en la figura 3. Los electrodos se ubicaron con una separación de $20 \mathrm{~mm}$ entre sí.

El sistema de acondicionamiento de señales EMG de la figura 2 se implementó mediante un hardware de acondicionamiento de las señales captadas por los electrodos EMG bipolares para dos canales (señal EMG1 y señal EMG2), compuesto de una primera etapa de preamplificación de ganancia 10; un filtro pasabanda con banda pasante entre 10 y $500 \mathrm{~Hz}$, que es el ancho de banda donde se concentra la información; un filtro tipo notch con frecuencia de corte de $60 \mathrm{~Hz}$ para reducir la interferencia electromagnética, una etapa de postamplificación con ganancia 5,7; una etapa que compensa offset DC de 0,8 V; y finalmente, un amplificador seguidor emisor, que entrega dos canales de señales EMG acondicionadas (señal canal EMG1 y señal canal EMG2) para acoplar las etapas de amplificación descritas anteriormente con la etapa de conversión analógica a digital del sistema de procesamiento en línea en el dsPIC.

El sistema de procesamiento en línea de la figura 2 utiliza el dsPIC33FJ256GP710 para: 1) digitalizar las señales de los canales EMG mediante su conversor análogo a digital de 12 bits, muestreando a una frecuencia de $10 \mathrm{kHz}, 2$ ) calcular en tiempo real las características del dominio del tiempo (etiqueta: 'Características', figura 2), y 3) clasificar el vector de características entregando una etiqueta que identifica la intención de movimiento (etiqueta: 'Intención de movimiento', figura 2). Los parámetros para el clasificador (etiqueta: 'Parámetros clasificador', figura 2) se obtienen luego de un procedimiento de entrenamiento fuera de línea que se programa en el dsPIC. 
Figura 3. Ubicación de los electrodos en el antebrazo: (a) canal l, (b) canal 2.

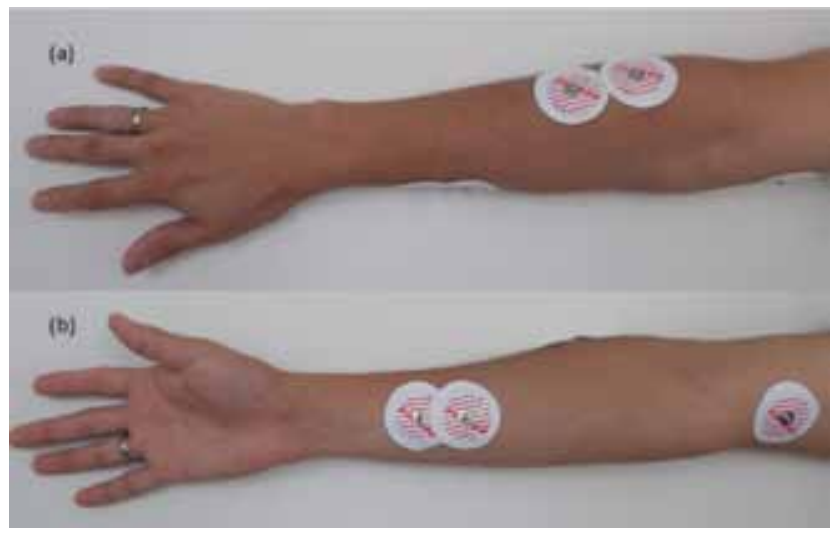

Fuente: presentación propia de los autores

\subsection{Software para detección de intención de movimiento}

La detección de intención de movimiento se logra mediante dos procedimientos diferenciados: la extracción de características desde las señales EMG y la clasificación de la intención de movimiento. Los algoritmos utilizados para estos procedimientos se describen a continuación.

\subsubsection{Extracción de características}

El éxito del control mioeléctrico de prótesis está fuertemente influenciado por la extracción de la información más relevante, necesaria y suficiente para discriminar con el mínimo de error los diferentes movimientos que se van a identificar en el sistema de prótesis. De los tres tipos de dominios para extracción de características -1) tiempo, 2) frecuencia, 3) tiempo y frecuencia-, las características que se obtienen sobre la base temporal se evalúan de forma más rápida. Phinyomark et al. [9] hacen una relación de hasta 50 tipos de características en el dominio del tiempo y la frecuencia, citando los artículos que describen cada una de ellas. En la tabla 1 se muestra una revisión de trabajos previos, teniendo en cuenta el porcentaje de éxito promedio, el número de clases que puede discriminar el clasificador, el número de canales de señales EMG utilizados, el tipo de características utilizadas, el tipo de clasificador y el tipo de procesamiento. Cipriani et al. [18] utilizan un clasificador basado en k-NN, y como característica de la señal EMG, el valor medio absoluto (MAV) para identificar seis clases con ocho canales EMG en personas con amputación; en dicho estudio se obtuvo una precisión del clasificador entre 80 y $96 \%$. 
Por su parte, Richard et al. [15] utilizaron clasificadores bayesianos y k-NN para comparar el desempeño. Identificaron quince clases haciendo uso de tres características (RMS, VAR, WL) de las señales EMG captadas por cuatro canales; en el caso del clasificador k-NN, obtuvieron una precisión de clasificación entre 94 y $95 \%$.

Tabla 1. Trabajos de control mioeléctrico

\begin{tabular}{|l|l|l|l|l|l|l|}
\hline \multicolumn{1}{|c|}{ Referencia } & $\begin{array}{c}\text { Porcentaje } \\
\text { de éxito } \\
\text { promedio }\end{array}$ & Clases & Canales & Características & Clasifıcador & $\begin{array}{c}\text { Tipo de } \\
\text { procesamiento }\end{array}$ \\
\hline $\begin{array}{l}\text { Phinyomark } \\
\text { et al., [9] }\end{array}$ & $93 \%$ & 11 & 4 & $\begin{array}{l}\text { SampEn, CC, } \\
\text { RMS, WL }\end{array}$ & LDA & Fuera de línea \\
\hline $\begin{array}{l}\text { Herle } \text { et al., } \\
\text { [11] }\end{array}$ & $91 \%-98 \%$ & 4 & 2 & AR & RNA & En línea \\
\hline $\begin{array}{l}\text { Khezri y } \\
\text { Jahed [12] }\end{array}$ & $95 \%-97 \%$ & 6 & 2 & $\begin{array}{l}\text { MAV, SSC, AR, } \\
\text { ZC, DWT }\end{array}$ & $\begin{array}{l}\text { Neuro- } \\
\text { Fuzzy }\end{array}$ & Tiempo real \\
\hline $\begin{array}{l}\text { Alkan y } \\
\text { Günay [13] }\end{array}$ & $96 \%-99 \%$ & 4 & 2 & MAV & LDA,SMV & Fuera de línea \\
\hline $\begin{array}{l}\text { Cipriani } \\
\text { et al., [18] }\end{array}$ & $80 \%-96 \%$ & 6 & 8 & MAV & k-NN & Tiempo real \\
\hline $\begin{array}{l}\text { Richard } \\
\text { et al., [15] }\end{array}$ & $94-95 \%$ & 15 & 4 & RMS, VAR, WL & $\begin{array}{l}\text { Bayesiano, } \\
\text { k-NN }\end{array}$ & Fuera de línea \\
\hline
\end{tabular}

En este estudio de investigación se consideraron solamente las características: valor medio absoluto (MAV), varianza (VAR) y raíz de la media al cuadrado (RMS). La característica MAV constituye una forma fácil de registrar los niveles de contracción del músculo y es muy popular en las aplicaciones de control mioeléctrico. El MAV de la señal se define así:

$$
M A V=\frac{1}{N} \sum_{n=1}^{N}\left|x_{n}\right|
$$

La característica VAR representa una medida de la potencia de la señal. Generalmente la varianza se define como 'el promedio de los valores al cuadrado de la desviación de la variable’. La varianza de la señal EMG puede ser definida así:

$$
V A R=\frac{1}{N-1} \sum_{n=1}^{N}\left|x_{n}^{2}\right|
$$


La característica RMS representa la amplitud modulada de un proceso aleatorio gaussiano que se relaciona con la fuerza constante y la contracción sin fatiga. La definición matemática de RMS se expresa así:

$$
R M S=\sqrt{\frac{1}{N} \sum_{n=1}^{N} x_{n}^{2}}
$$

En el estudio realizado por Phinyomark et al. [9], los sistemas que utilizaron las anteriores características arrojaron un porcentaje de acierto medio individual de $81,2 \%, 71,14 \%$ y $81,1 \%$; sin embargo, el criterio utilizado en este trabajo para la elección de este conjunto de características fue el de rapidez de ejecución. Para la extracción de características de la señal EMG, en este trabajo se escogió una ventana de 256 muestras con frecuencia de muestreo de $0,1 \mathrm{~ms}$, con lo que la ventana de análisis para extracción de características corresponde a un tiempo de $25 \mathrm{~ms}$, lo cual está dentro de la restricción de tiempo real, que debe ser menor a $300 \mathrm{~ms}$ [8].

\subsubsection{Clasificación de la intención de movimiento}

Las características extraídas se reúnen en un vector de características $v \in R^{3}$ conformado como $v=[M A V, V A R, R M S]$.

Aunque estas características se pueden calcular para los dos canales en la tarjeta de adecuación de señales EMG, en este trabajo se encontró que fue suficiente utilizar solo la información del canal 2 (asociado al músculo flexor superficial). Para cada uno de los cuatro tipos de movimiento que desean controlarse con la prótesis de mano, los vectores detectados se ubican alrededor de regiones distinguibles al representarse como puntos en el espacio euclidiano en $R^{3}$.

Identificar un movimiento consiste en clasificar un vector nuevo calculado, como perteneciente a un conjunto de clases posibles que definen grupos de vectores. La mayoría de las técnicas de agrupamiento desarrolladas para clasificar señales EMG se basan en adaptaciones de algoritmos generales de agrupación, como: k vecinos próximos (k-NN) [22], k-means [23], fuzzy c-means [24] y algoritmos jerárquicos [25].

En este trabajo se ha priorizado la obtención de intención de movimiento en tiempo real, por lo cual se propone un método simple de clasificación de los vectores, cuyo desarrollo puede dividirse en una etapa fuera de línea y una etapa en línea. 


\subsubsection{Etapa de procesamiento fuera de línea}

Consiste en obtener una base de datos a partir de experimentos de entrenamiento del clasificador, con ello se calculan los parámetros necesarios para el algoritmo de procesamiento en línea de clasificación de intenciones de movimiento en tiempo real.

Para este trabajo se obtuvieron las características para 40 movimientos por cada tipo de movimiento a identificar, conformando una base de datos de $M=160$ datos de características, 40 por cada clase de movimiento. Dicha base de datos fue previamente normalizada para concentrar los puntos en una región definida por una esfera de radio unitario. Los puntos pueden agruparse según la clase de movimiento, mediante $k$ esferas envolventes $E_{i}$ con centro en el centroide $C_{i}$, definido como la media matemática de los puntos pertenecientes a la clase $i$, para $i=1,2, \ldots, k$, y radio $r_{i}=\max _{k} d_{k}$, siendo $d_{k}=|| v_{k}-C_{i}||^{2} \mid v_{k} \hat{I} E_{i}$ la distancia euclidiana al cuadrado entre un vector $k$ perteneciente a la clase $i$ y el centroide $C_{i}$ de la clase. Este método de agrupación es conocido como $k$-means debe realizarse fuera de línea para todo el conjunto de datos. Para la aplicación presentada en este artículo, el número de grupos es $k=4$, ya que se conoce el número de clases que se va a identificar. Es común que existan regiones de intersección entre esferas envolventes, lo que dificulta la clasificación en línea de nuevos vectores.

En este trabajo se denomina $I_{u, v}=\left\{\left(v_{i}, c\right) \mid v_{i} \in E_{u} \cap E_{v}, i=1 . . m\right\}$ al conjunto de pares vector de características $v$ e identificador $c$ de la clase a la que pertenece, de la base de datos de entrenamiento y que están tanto en la esfera $E_{u}$ como en la esfera $E_{v}$.

La etapa fuera de línea se realiza en un computador personal adquiriendo las señales del canal vía puerto USB desde la tarjeta de adecuación de señales EMG. Con el software LabView se registran y se almacenan los datos en un archivo para conformar posteriormente los vectores de características; y mediante el software MatLab se calculan los centroides $C_{i}$ de las clases y el conjunto de vectores en las regiones de intersección de esferas $I_{u, v}$, que son los datos de salida de la etapa fuera de línea y que se deben programar sobre el sistema incrustado de la etapa en línea de tiempo real.

\subsubsection{Etapa de procesamiento en línea}

Durante esta etapa se realiza, en tiempo real, la clasificación de intención de movimiento para un nuevo vector de características calculado. Conocidos los centroides de las esferas envolventes, un nuevo vector obtenido en línea podría clasificarse encontrando el centroide más cercano al vector, pero habrá confusión de clases 
si el vector cae en una región de intersección entre esferas. Para solucionar este inconveniente existen muchas alternativas presentadas en la literatura; por ejemplo, clasificadores bayesianos [15] basados en redes neuronales, basados en técnicas de lógica difusa [12], o combinaciones de estos, pero la complejidad del clasificador resultante puede no ser práctica para aplicaciones en tiempo real.

En este trabajo se propone clasificar los vectores con el criterio de distancia a los centroides, lo cual constituye una operación computacionalmente eficiente. Así, un nuevo vector que no caiga dentro de regiones de intersección se clasifica en la clase donde la distancia desde el vector al centroide de esa clase sea la menor. Para aquellos vectores que caigan en regiones de intersección entre esferas envolventes, se aplica el algoritmo de clasificación k-NN convencional. Se asume que no se interceptan más de dos esferas en la base de datos de entrenamiento (lo cual debe ser verificado en la etapa fuera de línea).

El pseudocódigo mostrado en la tabla 2 muestra el algoritmo de clasificación para un nuevo vector.

Tabla 2. Pseudocódigo para el algoritmo de identificación de intención de movimiento

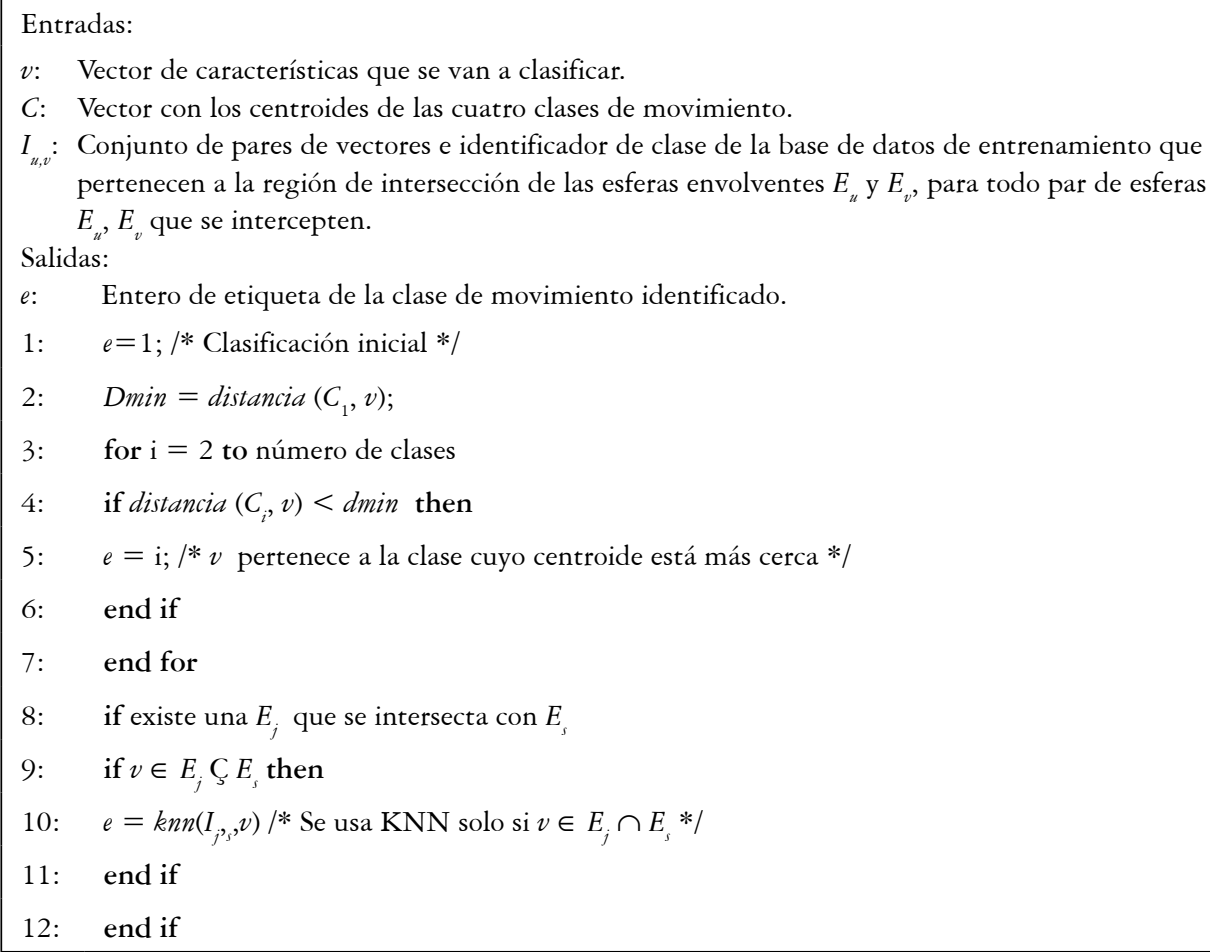

Fuente: presentación propia de los autores 
En la tabla 2 , la función distancia $\left(v_{1}, v_{2}\right)$ calcula la distancia euclidiana al cuadrado entre los vectores de entrada a la función. La función $k n n\left(D_{i}, j, v\right)$ devuelve un entero que identifica la clasificación del vector $v$ dentro del conjunto de datos de entrenamiento $D_{u, v}$. La tabla 3 muestra el pseudocódigo del algoritmo k-NN utilizado. Cabe resaltar que el clasificador utiliza dos etapas de clasificación: la primera está definida entre las líneas 1 a 7 del pseudocódigo de la tabla 2 y hace una clasificación inicial del nuevo vector basada solamente en las distancias a los centroides obtenidos en la etapa fuera de línea. La segunda etapa está descrita entre las líneas de código 8 a 12 en la tabla 2, y no se realiza siempre.

El aporte diferencial en este trabajo consiste en que el procesamiento que tiene costo computacional alto se realiza fuera de línea, dejando así un método simple de clasificación al procedimiento en línea, el cual usa k-NN solo cuando hay confusión entre dos grupos que en la fase fuera de línea hayan resultado muy próximos.

Tabla 3. Pseudocódigo para el algoritmo KNN

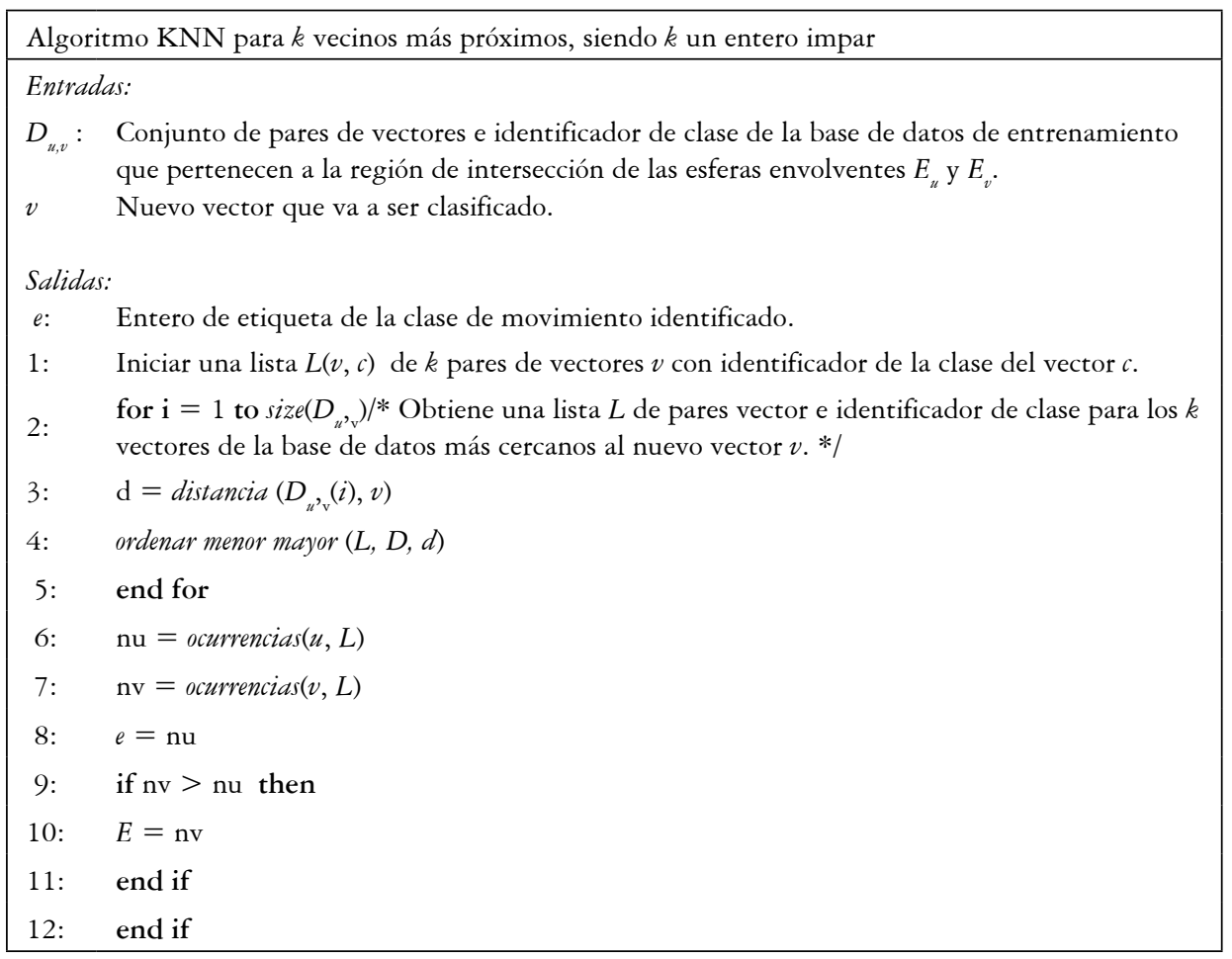

Fuente: presentación propia de los autores

Ing. Univ. Bogotá (Colombia), 19 (1): 27-50, enero-junio de 2015 
En la tabla 3, la función size $\left(D_{u, v}\right)$ obtiene el número de vectores de la lista de vectores, distancia $\left(v_{1}, v_{2}\right)$ y es la misma función de cálculo de distancia entre vectores descrita para la tabla 2. La función ordenar menor mayor $(L, D, d)$ ordena de menor a mayor la lista $L$ de los $k$ vectores más cercanos en $D$, según el criterio de menor distancia $d$ al nuevo vector $v$. La función ocurrencias $(i, L)$ obtiene el número de veces que ocurre la clase $i$ dentro de la lista $L$. Para este trabajo se utilizó el algoritmo k-NN con tres vecinos.

La desventaja del método k-NN es la necesidad de utilizar memoria en el sistema clasificador para guardar la información de las bases de datos de entrenamiento, pero en este trabajo esta desventaja se ve reducida por el hecho de que solo se deben guardar unos pocos vectores que queden ubicados en regiones de confusión, dado el caso particular donde la agrupación de los vectores de características favorece este enfoque.

\section{Configuración experimental}

Se pidió a cinco voluntarios (tres hombres y dos mujeres) sin amputación de mano que se ubicaran con el antebrazo extendido en una posición cómoda, como se muestra en la figura 4 , y se aplicó el siguiente protocolo de entrenamiento:

- En la fase de aprendizaje supervisado se le pidió a los participantes que mantuvieran su mano en posición de reposo (postura que se obtiene eliminando el offset de la señal EMG de cada participante) y que luego ejecutaran voluntariamente seis veces consecutivas los movimientos (abrir, pinza gruesa y agarre de poder) guiados por una señal auditiva generada por una aplicación en LabView; cada ensayo consistió en contraer el músculo durante 2 segundos y regresar a su estado de reposo durante otros 2 segundos. En esta fase de aprendizaje se pretende que el sujeto se familiarice con el sistema y que aprenda cómo controlar el prototipo de prótesis de mano.

- En la fase de entrenamiento se les pidió a los participantes que efectuaran los movimientos de cada primitiva de agarre en la secuencia: reposo, reposo-abrir, reposo-pinza gruesa y reposo-agarre de poder, en intervalos de 2 segundos, para la adquisición de las señales EMG (para entrenamiento y validación) necesarias para el procesamiento fuera de línea.

- En la fase de verificación, se les pidió a los participantes que efectuaran diez movimientos de cada postura de agarre durante 2 segundos en la secuencia dada en la fase de entrenamiento, dos veces al día (mañana y tarde) durante dos días, para un total de 40 movimientos por primitiva de agarre. En tiempo real en el sistema incrustado para cada acción del sujeto, se ejecutaron los 
algoritmos de extracción de características y de clasificación basados en distancia mínima y k-NN para obtener la intención de movimiento del sujeto. En línea estos comandos motores obtenidos por el clasificador fueron ejecutados en el prototipo de prótesis de mano.

Se registraron tanto la intención de movimiento calculada por el sistema, como la verdadera acción realizada por el sujeto.

Se define como medida de efectividad el porcentaje de éxito:

$$
\% \text { éxito }=\frac{\text { número de movimientos clasificados correctamente }}{\text { número total de movimientos clasificados }} \times 100
$$

La precisión del clasificador en la identificación de todas las clases de movimiento se promedia para calcular la precisión global del clasificador para un sujeto.

Figura 4. Montaje experimental

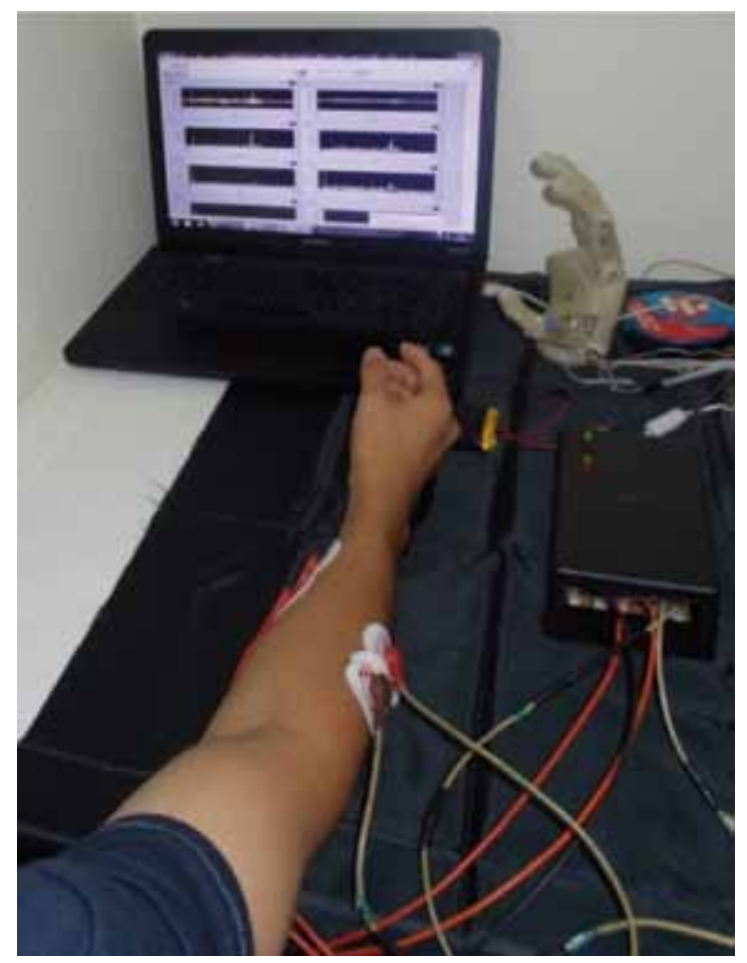

Fuente: presentación propia de los autores

Ing. Univ. Bogotá (Colombia), 19 (1): 27-50, enero-junio de 2015 


\section{Resultados}

En esta sección se presentan los resultados obtenidos de los métodos descritos en la sección 2 al realizar el montaje experimental descrito en la sección 3.

La figura 5 muestra el registro de los datos en LabView durante 4,5 segundos de los datos de la señal EMG para los músculos agonista y antagonista durante la ejecución de movimientos (mano en reposo, mano abierta, pinza gruesa y agarre de poder). Puede verse que para los cuatro movimientos realizados las señales acondicionadas permiten una diferenciación de los movimientos en el dominio del tiempo, lo cual es la base de las asunciones realizadas para la clasificación propuesta en este trabajo.

Figura 5. Señales EMG para diferentes movimientos

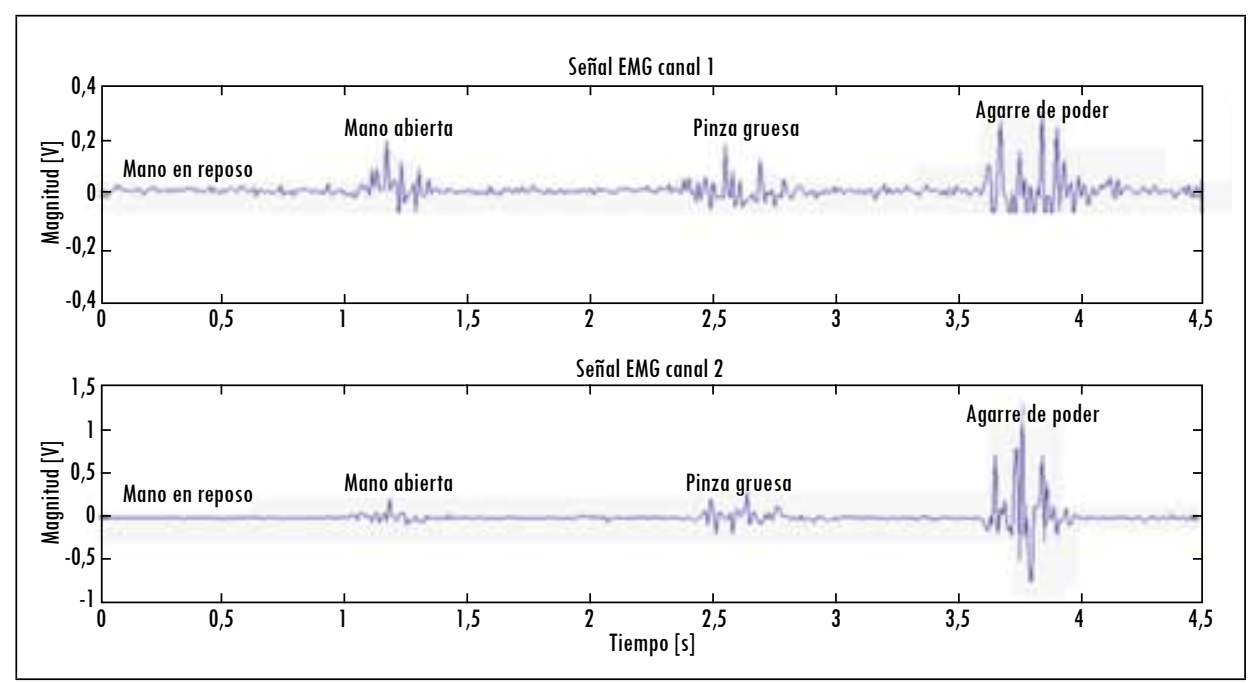

Fuente: presentación propia de los autores

En la figura 6a se muestra la separación entre clases lograda con las tres características descritas solo para el canal 1 (músculo extensor), y en la figura 6b, para solamente el canal 2 (músculo flexor). Se puede observar que no hay una buena separación de clases para los datos obtenidos para el canal 1 y que se presenta yuxtaposición de dos o más clases de movimientos, a diferencia del canal 2, que se puede separar en clases identificables. Esto demuestra, por una parte, que el método propuesto en este artículo requiere un proceso fuera de línea que permita identificar la ubicación óptima de electrodos para cada paciente de forma individual, proceso que se facilita gracias al esquema mostrado en la figura 2. 
Figura 6. Agrupación en clases de los vectores de entrenamiento según el centroide. a) Canal 1 (músculo extensor) y b) canal 2 (músculo flexor).
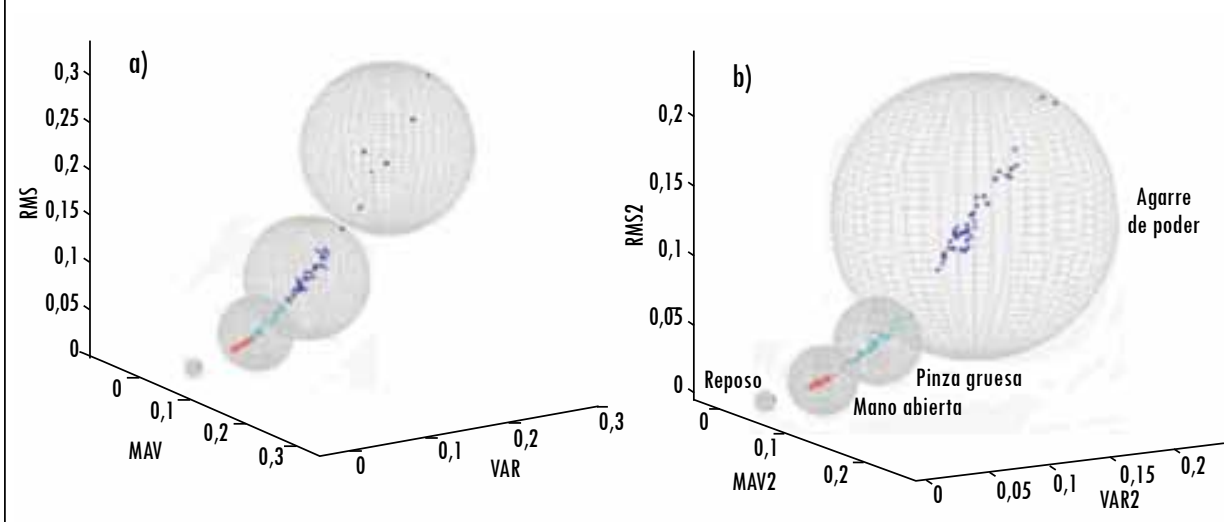

Fuente: presentación propia de los autores

Si la información obtenida de dos canales contribuyera a mejorar la separación entre un par de clases, el procedimiento de clasificación consistiría en conformar vectores con la información conjunta de los dos canales. Para los experimentos que se realizaron en personas con músculos sanos, la información de los dos canales no fue necesaria, por lo que se utilizó solamente la información de un solo canal, pero es muy probable que al realizar pruebas sobre pacientes con amputación se deba considerar la información de los dos canales.

Para poner en evidencia la efectividad de la variante de clasificador propuesta, en la tabla 4 se muestra la precisión del clasificador usando solo el cálculo de la distancia de la nueva muestra a los centroides de entrenamiento, para cuatro conjuntos de diez movimientos de cada primitiva motora efectuados por los cinco sujetos. Al calcular el porcentaje de éxito promedio se obtienen valores entre 75 y $87,5 \%$. El máximo error de clasificación se obtiene clasificando la intención de pinza gruesa, con cuatro aciertos de diez movimientos. Esto se explica porque algunos vectores de características caen en la región de intersección entre las clases abrir mano y pinza gruesa, como se muestra en la figura 7.

Al realizar el mismo experimento, pero incluyendo en el clasificador el algoritmo de k-NN para vectores que caen en las regiones de intersección de clases, se obtienen los porcentajes de éxito que se muestran en la tabla 5. Se observa un promedio de porcentaje de éxito que va del $85 \%$ al $92,5 \%$, y que es mayor a lo que se obtiene clasificando solo con el criterio de la distancia a los centroides. 
Figura 7. Intersección entre los grupos abrir mano y pinza gruesa

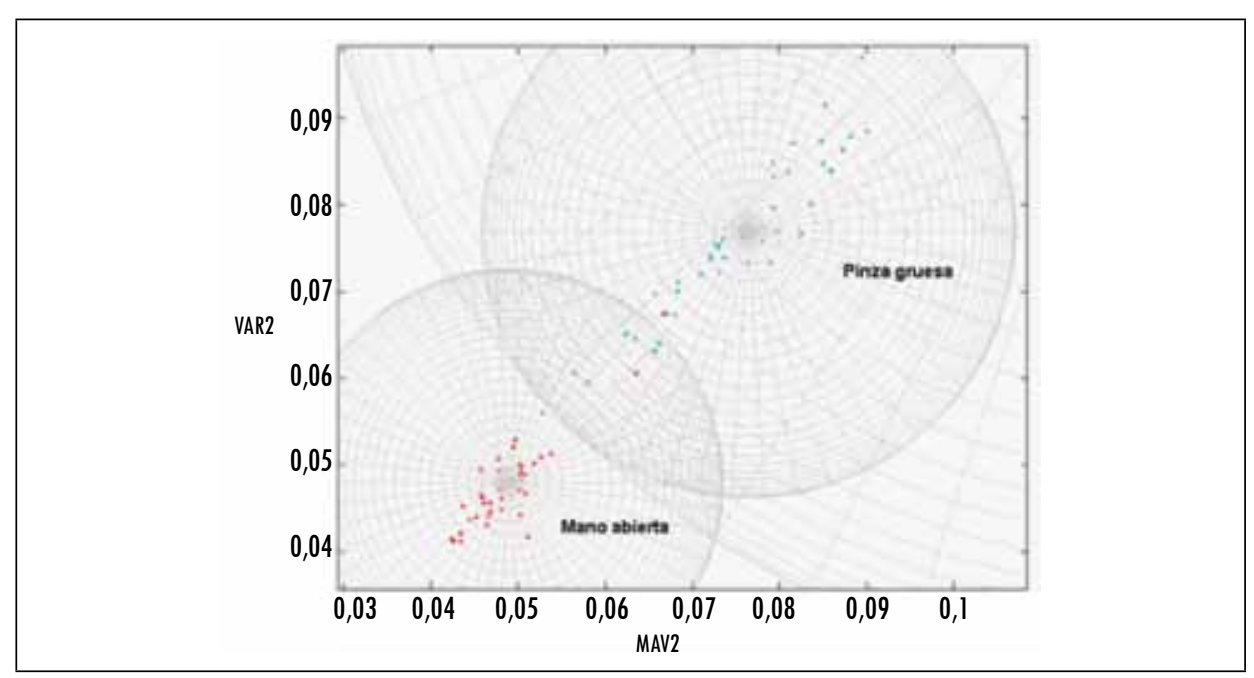

Fuente: presentación propia de los autores

Tabla 4. Porcentajes de éxito obtenidos en tiempo real utilizando clasifıcación basada solo en distancia al centroide

\begin{tabular}{|c|c|c|c|c|c|c|}
\hline Sujeto & $\begin{array}{c}\text { Conjunto } \\
\text { de ensayos }\end{array}$ & $\begin{array}{c}\text { Mano en } \\
\text { reposo }(\%)\end{array}$ & $\begin{array}{c}\text { Mano } \\
\text { abierta } \\
(\%)\end{array}$ & $\begin{array}{c}\text { Pinza } \\
\text { gruesa }(\%)\end{array}$ & $\begin{array}{l}\text { Agarre de } \\
\text { poder (\%) }\end{array}$ & $\begin{array}{c}\text { Porcentaje } \\
\text { de éxito } \\
\text { promedio }\end{array}$ \\
\hline \multirow{4}{*}{1} & 1 & 100 & 60 & 70 & 90 & 80,0 \\
\hline & 2 & 100 & 70 & 60 & 100 & 82,5 \\
\hline & 3 & 100 & 70 & 50 & 100 & 80,0 \\
\hline & 4 & 100 & 60 & 40 & 100 & 75,0 \\
\hline \multirow{4}{*}{2} & 1 & 100 & 70 & 60 & 100 & 82,5 \\
\hline & 2 & 100 & 70 & 60 & 90 & 80,0 \\
\hline & 3 & 100 & 60 & 70 & 100 & 82,5 \\
\hline & 4 & 100 & 70 & 50 & 100 & 80,0 \\
\hline \multirow{4}{*}{3} & 1 & 100 & 70 & 70 & 100 & 85,0 \\
\hline & 2 & 100 & 60 & 60 & 90 & 77,5 \\
\hline & 3 & 100 & 70 & 50 & 80 & 75,0 \\
\hline & 4 & 100 & 80 & 40 & 100 & 80,0 \\
\hline \multirow{4}{*}{4} & 1 & 100 & 60 & 60 & 100 & 80,0 \\
\hline & 2 & 100 & 70 & 50 & 90 & 77,5 \\
\hline & 3 & 100 & 70 & 60 & 100 & 82,5 \\
\hline & 4 & 100 & 50 & 70 & 90 & 77,5 \\
\hline
\end{tabular}




\begin{tabular}{|c|c|c|c|c|c|c|}
\hline Sujeto & $\begin{array}{c}\text { Conjunto } \\
\text { de ensayos }\end{array}$ & $\begin{array}{c}\text { Mano en } \\
\text { reposo (\%) }\end{array}$ & $\begin{array}{c}\text { Mano } \\
\text { abierta } \\
(\%)\end{array}$ & $\begin{array}{c}\text { Pinza } \\
\text { gruesa (\%) }\end{array}$ & $\begin{array}{c}\text { Agarre de } \\
\text { poder (\%) }\end{array}$ & $\begin{array}{c}\text { Porcentaje } \\
\text { de éxito } \\
\text { promedio }\end{array}$ \\
\hline \multirow{3}{*}{5} & 1 & 100 & 60 & 80 & 100 & 85,0 \\
\cline { 2 - 7 } & 2 & 100 & 70 & 60 & 100 & 82,5 \\
\cline { 2 - 7 } & 3 & 100 & 60 & 50 & 90 & 75,0 \\
\cline { 2 - 7 } & 4 & 100 & 80 & 70 & 100 & 87,5 \\
\hline
\end{tabular}

Fuente: presentación propia de los autores

Tabla 5. Porcentajes de éxito obtenidos en tiempo real utilizando el clasificador basado en la distancia mínima y el clasificador KNN para muestras en la intersección de clases

\begin{tabular}{|c|c|c|c|c|c|c|}
\hline Sujeto & $\begin{array}{c}\text { Conjunto } \\
\text { de ensayos }\end{array}$ & $\begin{array}{c}\text { Mano en } \\
\text { reposo (\%) }\end{array}$ & $\begin{array}{c}\text { Mano } \\
\text { abierta (\%) }\end{array}$ & $\begin{array}{c}\text { Pinza } \\
\text { gruesa }(\%)\end{array}$ & $\begin{array}{l}\text { Agarre de } \\
\text { poder (\%) }\end{array}$ & $\begin{array}{c}\text { Porcentaje } \\
\text { de éxito } \\
\text { promedio }\end{array}$ \\
\hline \multirow{4}{*}{1} & 1 & 100 & 80 & 70 & 100 & 87,5 \\
\hline & 2 & 100 & 90 & 70 & 100 & 90,0 \\
\hline & 3 & 100 & 90 & 60 & 100 & 87,5 \\
\hline & 4 & 100 & 80 & 80 & 100 & 90,0 \\
\hline \multirow{4}{*}{2} & 1 & 100 & 80 & 80 & 100 & 90,0 \\
\hline & 2 & 100 & 90 & 60 & 90 & 85,0 \\
\hline & 3 & 100 & 100 & 70 & 100 & 92,5 \\
\hline & 4 & 100 & 80 & 80 & 100 & 90,0 \\
\hline \multirow{4}{*}{3} & 1 & 100 & 90 & 80 & 100 & 92,5 \\
\hline & 2 & 100 & 90 & 70 & 100 & 90,0 \\
\hline & 3 & 100 & 80 & 70 & 100 & 87,5 \\
\hline & 4 & 100 & 100 & 80 & 100 & 95,0 \\
\hline \multirow{4}{*}{4} & 1 & 100 & 80 & 70 & 100 & 87,5 \\
\hline & 2 & 100 & 80 & 80 & 90 & 87,5 \\
\hline & 3 & 100 & 90 & 70 & 100 & 90,0 \\
\hline & 4 & 100 & 80 & 70 & 90 & 85,0 \\
\hline \multirow{4}{*}{5} & 1 & 100 & 100 & 80 & 100 & 95,0 \\
\hline & 2 & 100 & 90 & 80 & 100 & 92,5 \\
\hline & 3 & 100 & 90 & 70 & 100 & 90,0 \\
\hline & 4 & 100 & 80 & 80 & 100 & 90,0 \\
\hline
\end{tabular}

Fuente: presentación propia de los autores 
Al comparar los resultados experimentales del sistema de identificación de intención de movimiento propuesto (consignados en la tabla 5), con los resultados obtenidos en los trabajos mencionados en la tabla 1, es claro que el sistema propuesto en este trabajo es una buena alternativa para clasificar la intención de movimiento a partir de señales EMG.

En cuanto al tiempo de procesamiento y el consumo de recursos computacionales, se obtiene que usando un dsPIC a una frecuencia de $40 \mathrm{MHz}$, el tiempo requerido por los algoritmos de extracción de características, clasificación de distancia mínima al centroide y KNN para obtener una intención de movimiento es de $31 \mathrm{~ms}$. El espacio de memoria usada es de 3 Kbytes de memoria RAM y 3,9 Kbytes de memoria de programa flash, con lo que el sistema propuesto resulta adecuado para el propósito de control de una prótesis de mano activa.

\section{Conclusiones}

En este trabajo se propuso y se desarrolló un sistema de detección de intención de movimiento a partir de señales EMG captadas del músculo flexor superficial del antebrazo de una persona sin amputación de mano. Se llevaron a cabo experimentos en tiempo real utilizando el sistema desarrollado, y los resultados obtenidos muestran que, gracias a un adecuado hardware de adquisición y al método de clasificación propuesto, es posible lograr una alta tasa de aciertos en el reconocimiento de intención de movimiento.

El sistema demuestra ser efectivo y útil para detección en tiempo real, obteniendo un porcentaje de éxito de clasificación entre el 85 y $92,5 \%$, aunque la eficiencia del clasificador depende de una buena separación entre las clases. Para el logro de una buena separabilidad, debe tenerse en cuenta: la ubicación de los sensores EMG, el grupo de características temporales utilizadas, el tipo de movimientos que se van a clasificar y el sistema de adquisición y procesamiento utilizado.

La extensión del enfoque a otras características u otros músculos desde donde se capten las señales dependerá de un análisis para determinar si la separabilidad de las clases admite el enfoque presentado en este trabajo.

Con base en la alta precisión del clasificador, es posible generar comandos motores para mover una mano artificial con diferentes posturas de agarre. El sistema de adquisición y de procesamiento de señales EMG desarrollado resulta portable, gracias a estar soportado por un dsPIC, lo cual lo hace de bajo costo y de gran flexibilidad.

El sistema implementado puede ser extrapolado a pacientes con amputación de mano que hayan tenido un previo entrenamiento de rehabilitación y que 
puedan generar actividad mioeléctrica en su miembro residual. El sistema también podría ser utilizado para gobernar cualquier mano activa cuya estrategia de control permita recibir intención de movimiento captada desde una persona y generar comandos motores para realizar hasta cuatro posturas de agarre.

Como trabajos futuros se han definido: realizar una evaluación más detallada acerca de la mejor combinación de características que se van a utilizar con base en el tiempo real y el porcentaje de acierto; pasar el sistema a un hardware de montaje superficial que pueda ser incrustado en la palma de la prótesis de mano, y evaluar el desempeño del sistema cuando sea utilizado por pacientes con amputación de mano.

\section{Agradecimientos}

Los autores agradecen a la Universidad del Cauca por el soporte brindado a la presente investigación mediante infraestructura, equipos y facilidades para la dedicación de tiempo del recurso humano.

\section{Referencias}

[1] Shadow Robot Company. (2014). [En línea]. Disponible en: http://www.shadowrobot. com/products/ dexterous-hand/.

[2] RSLSteeper. (s. f.). [En línea]. Disponible en: http://es.bebionic.com/the_hand.

[3] C. Cipriani et al., "The effects of weight and inertia of the prosthesis on the Sensitivity of EMG pattern recognition in relax state," Journal Prosthetics Orthotics, vol. 24, no. 2, pp. 86-92, 2012.

[4] K. Ernest, S. Erik y E. Kevin, "Combined surface and intramuscular EMG for improved real-time myoelectric control performance," Biomedical Sigmal Processing Control, vol. 10, pp. 102-107, mar. 2014.

[5] K. Englehart y B. Hudgins, "A robust, real-time control scheme for multifunction myoelectric control," IEEE Transactions Biomedical Engineering, vol. 50, pp.848-854, jul. 2003.

[6] E. N. Kamavuako et al., "Estimation of grasping force from features of intramuscular EMG signals with mirrored bilateral training," Annals Biomedical Engineering, vol. 40, pp. 648-656, mar. 2012.

[7] R. Clement, K. Bugler y C. Oliver, "Bionic prosthetic hands: A review of present technology and future aspirations," The Surgeon, vol. 9, no. 6, pp. 336-340, 2011.

[8] A. Phinyomark, C. Limsakul y P. Phukpattaranot, "A novel feature extraction for robust EMG pattern recognition," Journal Computing, vol. 1, no. 1, pp. 71-80, dic. 2009.

[9] A. Phinyomark et al., "EMG feature evaluation for improving myoelectric pattern recognition robustness," Expert Systems Applications, vol. 40, no. 12, pp. 4832-4840, sep. 2013. 
[10] E. Shroffe y P. Manimegalai, "Hand gesture recognition based on EMG signal using ANN," International Journal Computer Application, vol. 2, no. 3, pp. 31-39, abr. 2013.

[11] S. Herle et al., "Myoelectric control strategies for a human upper limb prosthesis," Journal Control Engineering Applied Informatics, vol. 14, no. 1, pp. 58-66, 2012.

[12] M. Khezri y M, Jahed. (Dic. 2007). "Real-time intelligent pattern recognition algorithm for surface EMG signals," BioMedical Engineering. [En línea]. Disponible en: http://www. biomedical-engineering-online.com/content/6/1/45.

[13] A. Alkan y M. Günay, "Identification of EMG signals using discriminant analysis and SVM classifier”. Expert System Applications, vol. 39, no. 1, pp. 44-47, 2012.

[14] A. Subasi, "Classification of EMG signals using PSO optimized SVM for diagnosis of neuromuscular disorders," Computers Biology Medicine, vol. 43, no. 5, pp. 576-586, jun. 2013.

[15] R. Richard et al., "Towards sEMG classification based on Bayesian and k-NN to control a prosthetic hand," Biosignals Biorobotics Conference, pp. 1-6, feb. 2013.

[16] P. Parker, K. Englehart y B. Hudgins, "Myoelectric signal processing for control of powered limb prostheses," Journal Electromyography Kinesiology, vol. 16, no. 6, pp. 541-548, dic. 2006.

[17] N. Jiang, K. Englehart y P. Parker, "Extracting simultaneous and proportional neural control information for Multiple-DOF prostheses from the surface electromyographic signal," IEEE Transactions Biomedical Engineering, vol. 56, no. 4, pp. 1070-1080, abr. 2009.

[18] C. Cipriani et al. "Online myoelectric control of a dexterous hand prosthesis by transradial amputees”, IEEE Transactions Neural Systems Rehabilitation Engineering, vol. 19, no. 3, pp. 260-270, jun. 2011.

[19] F. Tenore et al., "Decoding of individuated finger movements using surface electromyography," IEEE Transactions Biomedical Engineering, vol. 56, no. 5, pp. 1427-1434, may. 2009.

[20] C. Quinayás et al., "Diseño y construcción de la prótesis robótica de mano UC-1," Ingeniería Universidad, vol.14, no. 2, pp. 223-237, 2010.

[21] R. Merletti y P. Parker. Electromyography: physiology, engineering and noninvasive applications. Wiley Online Library, 2005.

[22] K. Kim et al., "Comparison of K-nearest neighbor, quadratic discriminant and linear discriminant analysis in classification of electromyogram signals based on the wrist-motion directions," Current Applied Physics, vol. 11, no. 3, pp. 740-745, may. 2011.

[23] C. D. Katsis et al., "A novel method for automated EMG decomposition and MUAP classification," Artificial Intelligence Medicine, vol. 37, no. 1, pp. 55-64, may. 2006.

[24] A. Ajibove y R. Weir, "A heuristic fuzzy logic approach to EMG pattern recognition for multifunctional prosthesis control," IEEE Transaction Neural System Rehabilitation Engineering, vol. 13, no. 3, pp. 280-291, sep. 2005. 
50 César Augusto Quinayás-Burgos, Carlos Alberto Gaviria-López

[25] M. Okamoto y Y. Matsubara, "EMG pattern classification using hierarchical network based on boosting approach," International Journal Innovative Computing, Information Control, vol. 5, no. 12(B), pp. 4935-4943, dic. 2009. 